\title{
Recuento de los eventos artístico culturales del Departamento de Bellas Artes de la Universidad de Sonora
}

\author{
Gustavo Chaidez \\ Licenciatura es Artes Escénica \\ Ivanna Gómez \\ Licenciatura en Música
}
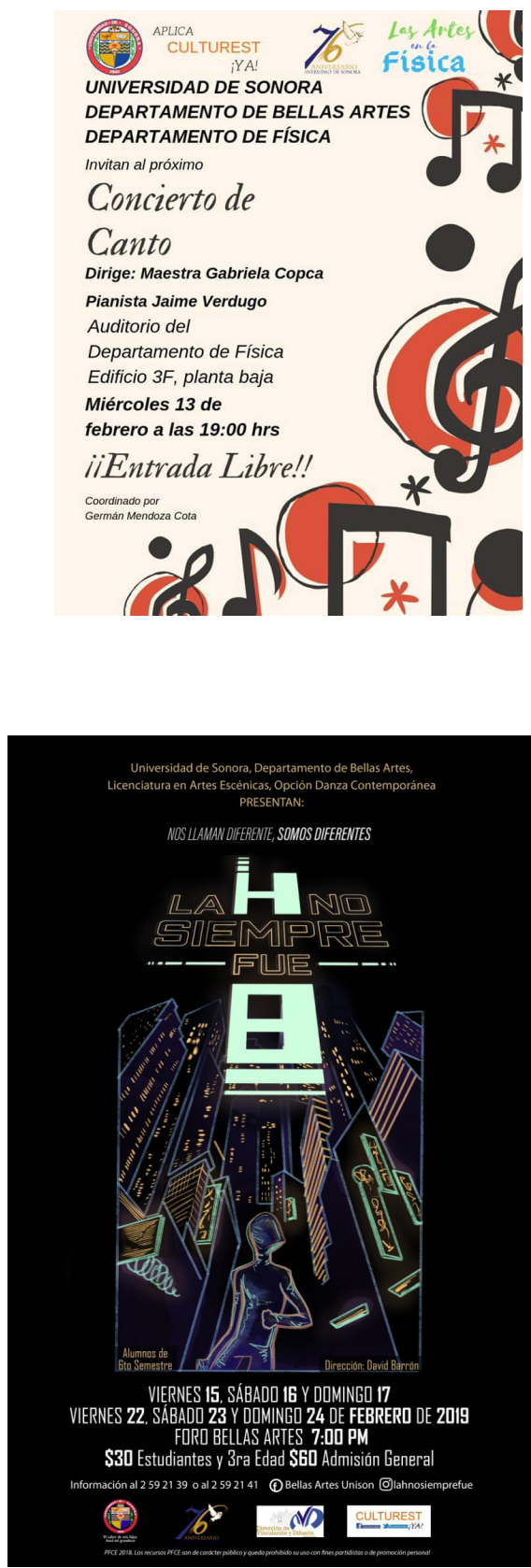

El siguiente texto muestra, a manera de resumen, todos los eventos culturales que tuvieron lugar en el transcurso del semestre 2019-1, enero-mayo, en colaboración con la Universidad de Sonora ya sea en las mismas instalaciones o por participación de profesorado o alumnos del Alma Mater.

\section{6 de febrero: Los padres terribles}

Obra de teatro de los alumnos de $8^{\text {vo }}$ semestre de la licenciatura en Artes Escénicas, opción actuación, dirigida por el maestro Marcos González Navarro. Presentada en el Foro de Bellas Artes de la UNISON con varias temporadas entre febrero y abril.

\section{5 de febrero: La $\mathrm{H}$ no siempre fue 8}

Coreografía de los alumnos de $6^{\text {to }}$ semestre de la licenciatura en Artes Escénicas, opción danza, dirigida por el maestro David Barrón. Presentada en el Foro de Bellas Artes de la UNISON del 15 al 24 de febrero.

18 de febrero: Laboratorio de investigación-creación (de la acción a la representación).

Taller teórico-práctico sobre la investigación de la danza. Impartido por Hilda Islas como parte del IV Congreso Nacional sobre Educación Superior en las Artes de la UNISON los días 18 y 19 de febrero.

20 de febrero: Arte, Cibercultura y Sociedad contemporánea. Una aproximación de nuevos paradigmas entre la sociedad y el arte en nuestros días Plática impartida por el Dr. Álvaro Díaz Rodríguez. Durante la conferencia magistral, parte del IV Congreso Nacional sobre Educación Superior en las Artes, el Dr. Álvaro Díaz expuso el poder de la tecnología dentro del área artística, sobresaliendo la parte musical. Evento abierto al público en general, siendo miembros del área artística los mayores asistentes.

20 de febrero: Las emociones y sentimientos en el proceso de creación de un texto musical de tradición occidental

En esta ponencia el Dr. Jesús Eduardo Ramírez Paredes expone como duda central si la música significa algo, a través de herramientas generadas por varias disciplinas como la filosofía, psicología y semiótica se establece que en base a emociones, sensaciones y sentimientos el oyente puede entender el significado de la música la mayor parte del tiempo. 
20 de febrero: Análisis de la condición Sinestésica y su relación auditiva-visual en el proceso compositivo de Rimski- Korsakov

El egresado de la licenciatura en música Román Alexis López Cañez explica el funcionamiento del sistema de Rimski-Korsakov, que hace uso de la sinestesia, mezclando las notas con colores y cuestiones mentales, para exponer el funcionamiento del sistema se utilizó la partitura de Scheherezade.

\section{2 de febrero: Sewa Tomteme, tecnología aplicada para las artes}

Conferencia por parte del Maestro. Francisco Ramírez Arroyo. Parte del IV Congreso Nacional sobre Educación Superior en las Artes en Foro de Bellas Artes de la Universidad de Sonora. Presentación del proyecto Sewa Tomteme, escuela artística con plan especial para la comunidad Yaqui, incorporando herramientas tecnológicas para su desarrollo.

\section{1 de marzo: Capos del Sabor Vol. 2 Caro Quinteto}

"Tocada" del grupo Caro Quinteto en el Bar London Pub. Con miembros de la Licenciatura en Música, el conjunto presenta una serie de arreglos creados por él fundado Martin Caro, donde el género de norteño y ritmos latinos se fusionan para crear esta propuesta musical.

\section{1 de marzo: VISO Out Festival 2019}

Festival artístico alternativo realizado del 1 al 3 de marzo en Quinta de Anza donde participaron varios alumnos, maestros y egresados de la licenciatura en Artes Escénicas con obras de danza y teatro.

\section{2 de marzo: La Huelga}

Monólogo presentado por Laisha Centeno, alumna de la licenciatura en Artes Escénicas, en Casa Andamios los días 12 y 13 de abril.

\section{1 de marzo: Concierto a cielo abierto Orquesta Los Cacha}

Concierto de salsa y otros ritmos latinos en la Plaza del Estudiante dentro de la Universidad de Sonora como parte del marco del Festival de Primavera 2019. Un evento que contó con la participación de la Orquesta Los Cacha de Juan Ayala, integrada por gran parte de alumnos de la Universidad, invitando a un público variado a bailar a cielo abierto.

\section{2 de marzo: Viernes de concierto- área de piano}

En esta participación se observó un programa variado en el que se interpretaron obras de compositores como Bach, Ruiz Armengol, Telemann, Haydn, Debussy, Beethoven, Chopin y Clementi, , los alumnos participantes fueron: Luis Arturo Silva Molina, Daniel Isaac Ruiz Moreno, Alfredo Pompa Dorado, Cesar Gustavo Ledezma Sáenz, Teresita Ibarra, Julio Cesar Orci Moreno, Andrea Susette Navarro Save, Cesar Daniel Quiroz Flores, Luz Amelia Martínez Martínez y Luz Elena Martínez Martínez.
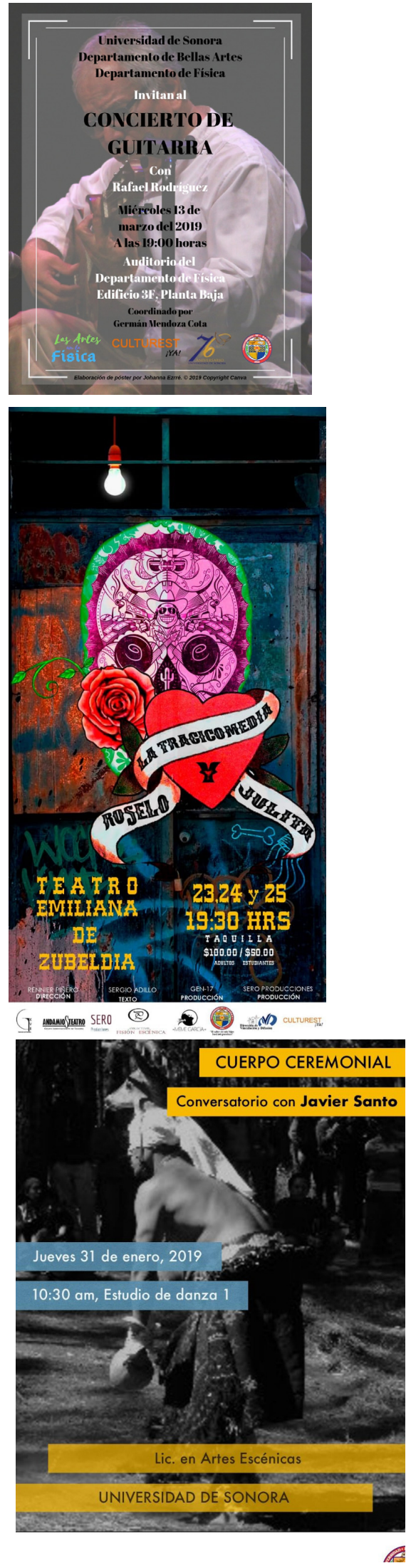
26 de marzo: Ejecutor 14

Monólogo presentado por Nicolás Rivera, egresado y profesor de la licenciatura en Artes Escénicas, bajo la dirección de Marcos González. Evento realizado del 26 al 29 de marzo en Quinta de Anza.

\section{3 de abril: Impropio ser alienante}

Coreografía presentada por David Barrón, profesor de la licenciatura en Artes Escénicas, junto con otros profesores y un alumno. Obra exhibida como parte de la temporada de primavera de teatro El Mentidero.

\section{5 de abril: Pinocho/Alicia en el país de las maravillas}

Teatro infantil presentado por alumnos de la materia de Voz II de la licenciatura en Artes Escénicas. Tuvo lugar en el patio de Letras y Lingüística de la UNISON el día 15 de abril y estuvo bajo la coordinación del maestro Luis Ricardo Gaitán.

\section{9 de abril: Los concheros que me tocan}

Coreografía dirigida por David Barrón, presentada durante el festival Un Desierto Para la Danza 2019. En esta obra participaron, entre otros profesionales, los alumnos Ramsés Carranco y Emiliano Castro junto con la profesora Perla López de la licenciatura en Artes Escénicas, opción danza contemporánea. Ocurrió el día 19 de abril en el Teatro de la Ciudad de la Casa de la Cultura.

\section{1 de mayo: Master Class por Martin Caro "Percusión dentro del Jazz Latino"}

Taller de ritmos de Jazz Latino impartido por Martín Caro, maestro en percusión, por parte de la primera edición de Solaría Festival de Jazz, en el Restaurarte Está Cabral. Evento en el que, acompañado de músicos egresados de la Licenciatura en Música de la Universidad de Sonora, se presentaron diferentes ritmos bases para la ejecución del Jazz Latino.

\section{1 de mayo: Concierto de Jazz por Alejandro Corona. Tributo a Bill Evans}

Concierto a cielo abierto en el Restaurarte Está Cabral por parte del maestro de la Licenciatura en Música Alejandro Corona. Haciendo un tributo a Bill Evans, se presentó una variedad de canciones y poemas, con la voz de la maestra de la Universidad de Sonora Glenda Landavazo y músicos de la misma Alma Mater.
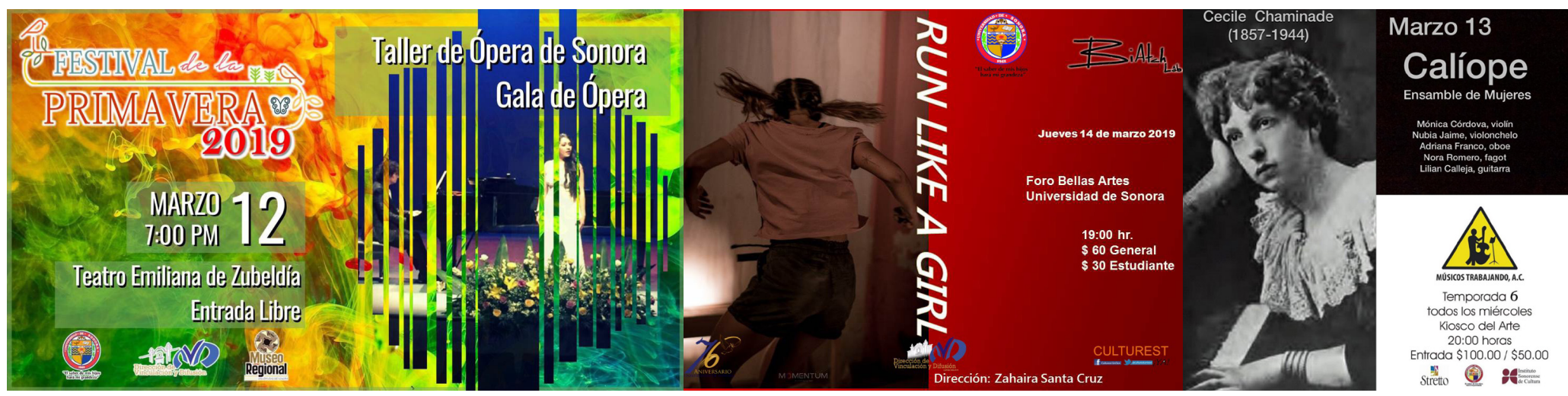

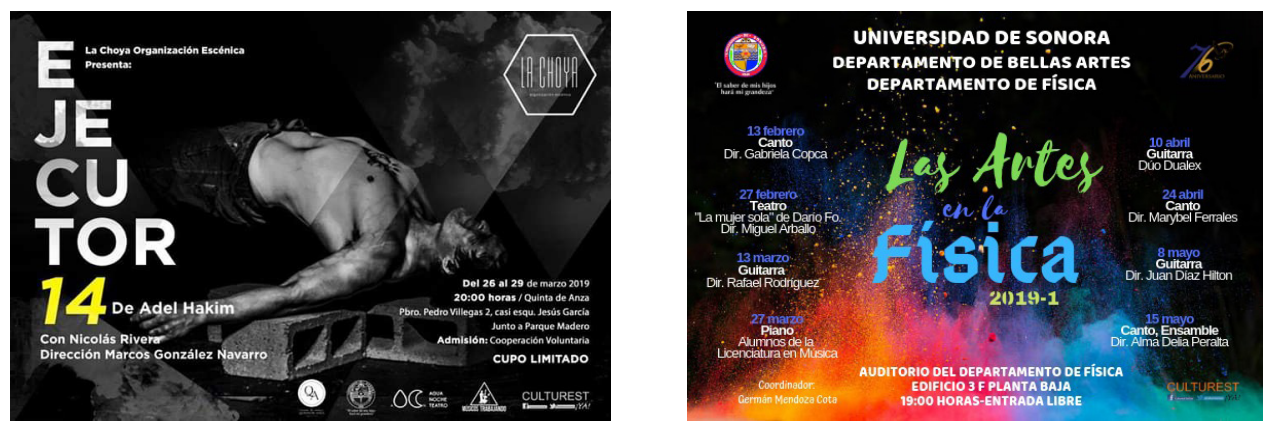

\section{2 de mayo: Los buenos manejos}

Divertida obra de teatro presentada por alumnos de $4^{\text {to }}$ semestre de la licenciatura en Artes Escénicas bajo la dirección de la maestra Adria Peña. Presentada en el Foro de Bellas Artes de la UNISON del 2 al 5 de mayo.

4 de mayo: Solistas de la Orquesta de guitarras de Sonora

Concierto con un programa extenso y variado en el que se presentó un repertorio de obras extensas de varios movimientos. Los alumnos participantes fueron Roberto Soto, Carlos Martínez, Marco Lamadrid, Kevin Felix, Juan Carlos Felix, Alejandro Vega e Iván Durán

\section{8 de mayo: Presentación de la película "Mis demonios nunca} juraron soledad". $7^{\text {mo }}$ FICH

"Mis demonios nunca juraron soledad" fue la película con la que inició esta $7^{\mathrm{ma}}$ edición del Festival Internacional de Cine en Hermosillo, teniendo como sede la sala del cine Cinemex Metrocentro. Es la primera cinematografía en la que el alumno egresado de la Licenciatura en Música Alejandro Karo participa, siendo de su propia creación toda música presentada en la cinta.

\section{8 de mayo: Las artes en la física. Concierto de guitarra}

Las participaciones del área de Bellas Artes en el Departamento de Física terminaron esta temporada con un concierto de guitarra clásica, Participaron 4 alumnos de la Licenciatura en Música del área de guitarra Juan Carlos Félix Córdova, David Barroso, Cesar Antonio Quijada Amavizca y Alejandro Vega Bustamante.

\section{0 de mayo: Presentación de la obra "Versión inmaterial"}

Coreografía de los alumnos de $6^{\text {to }}$ semestre de la licenciatura en Artes Escénicas, opción danza contemporánea, presentada en el Foro de Bellas Artes del 10 al 12 de mayo bajo la dirección de David Barrón.

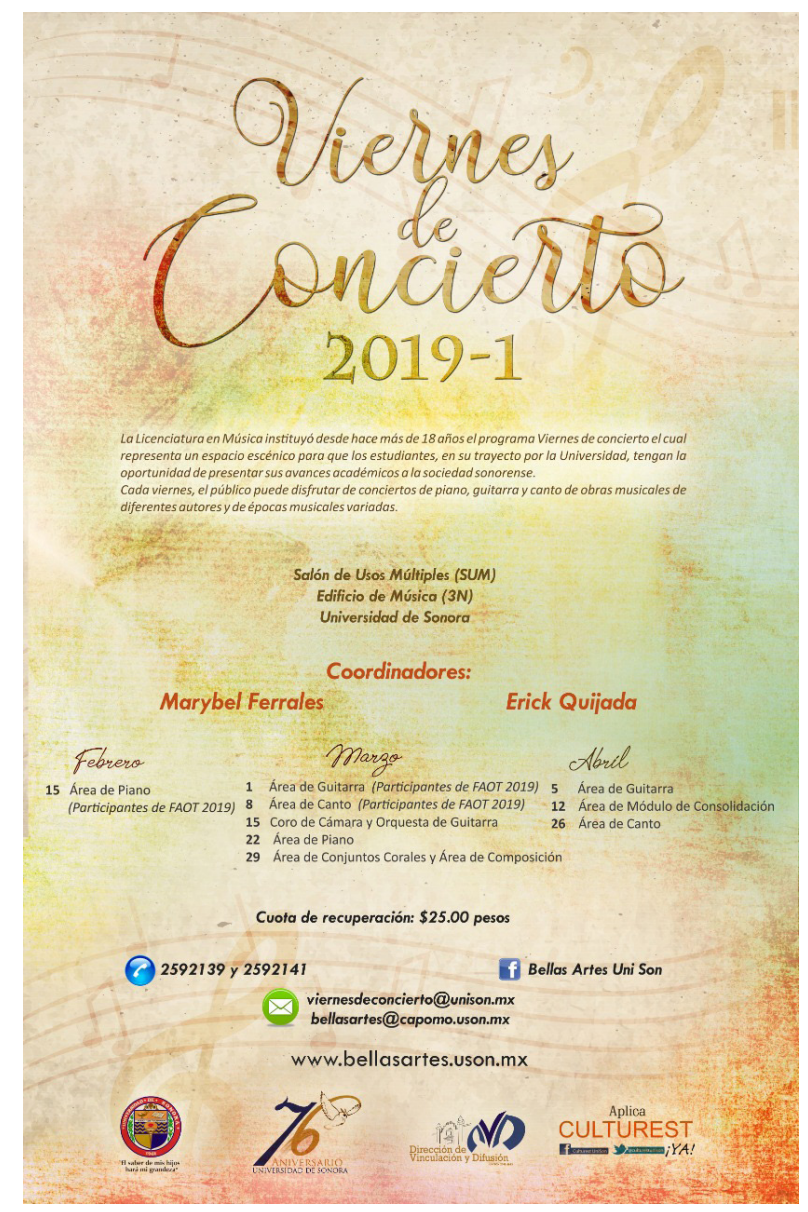

